\title{
Relative difference among 27 functional measures in patients with knee osteoarthritis: an exploratory cross-sectional case-control study
}

\author{
K. Vårbakken ${ }^{1,2^{*}}$, H. Lorås ${ }^{3}$, K. G. Nilsson ${ }^{4}$, M. Engdal $\left.\right|^{5}$ and A. K. Stensdotter ${ }^{1,2}$
}

\begin{abstract}
Background: To raise the effectiveness of interventions, clinicians should evaluate important biopsychosocial aspects of the patient's situation. There is limited knowledge of which factors according to the International Classification of Function, Disability, and Health (ICF) are most deviant between patients with knee osteoarthritis $(\mathrm{KOA})$ and healthy individuals. To assist in measures' selection, we aimed to quantify the differences between patients with KOA and healthy controls on various measures across the ICF dimensions of body function, activity, and participation.
\end{abstract}

Methods: We performed an exploratory cross-sectional case-control study. In total, 28 patients with mild-tomoderate KOA (mean age 61 years, 64\% women) referred by general physicians to a hospital's osteoarthritis-school, and 31 healthy participants (mean age 55 years, 52\% women), volunteered. We compared between-group differences on 27 physical and self-reported measures derived from treatment guidelines, trial recommendations, and trial/outcome reviews. Independent t-test, Chi-square, and Mann-Whitney $U$ test evaluated the significance for continuous parametric, dichotomous, and ordinal data, respectively. For parametric data, effect sizes were calculated as Cohen's $d$. For non-parametric data, $d s$ were estimated by $p$-values and sample sizes according to statistical formulas. Finally, all $d s$ were ranked and interpreted after Hopkins' scale. An age-adjusted sensitivity-analysis on parametric data validated those conclusions.

Results: Very large differences between patients and controls were found on the Pain numeric rating scale ${ }^{1}$, the Knee Injury and Osteoarthritis Scale (KOOS, all subscales) ${ }^{2}$, as well as the Örebro Musculoskeletal psychosocial scale ${ }^{3}$ $(P<0.0001)$. Large differences were found on the Timed 10-steps-up-and-down stair climb test ${ }^{4}$ and Accelerometer registered vigorous-intensity physical activity in daily life $(P<0.001)$. Respectively, these measures clustered on ICF as follows: ${ }^{1}$ body function, ${ }^{2}$ all three ICF-dimensions, ${ }^{3}$ body function and participation, ${ }^{4}$ activity, and ${ }^{5}$ participation.

Limitations: The limited sample excluded elderly patients with severe obesity.

\footnotetext{
* Correspondence: kjartanv@ntnu.no

'Department of Neuromedicine and Movement Science, Norwegian

University of Science and Technology, Trondheim, Norway

${ }^{2}$ Faculty of Medicine and Health Sciences, NTNU, Health og Social building,

7491 Trondheim, Norway

Full list of author information is available at the end of the article
}

(c) The Author(s). 2019 Open Access This article is distributed under the terms of the Creative Commons Attribution 4.0 International License (http://creativecommons.org/licenses/by/4.0/), which permits unrestricted use, distribution, and reproduction in any medium, provided you give appropriate credit to the original author(s) and the source, provide a link to the Creative Commons license, and indicate if changes were made. The Creative Commons Public Domain Dedication waiver (http://creativecommons.org/publicdomain/zero/1.0/) applies to the data made available in this article, unless otherwise stated. 
(Continued from previous page)

Conclusions: Very large differences across all ICF dimensions were indicated for the KOOS and Örebro questionnaires together for patients aged 45-70 with KOA. Clinicians are suggested to use them as means of selecting supplementary measures with appropriate discriminative characteristics and clear links to effective therapy. Confirmative studies are needed to further validate these explorative and partly age-unadjusted conclusions.

Keywords: Osteoarthritis, knee, Muscle strength dynamometer, Physical fitness, Physical examination, Physical activity, Exercise, Activities of daily living, International classification of functioning, disability, and health, Psychology, Sociological factors

\section{Background}

Osteoarthritis is the second most prevalent condition of all musculoskeletal and rheumatic diseases and the main contributor to social activity limitations $[1,2]$. Knee osteoarthritis (KOA) has an incidence of 240 per 100, 000 person-years in adults, which is more than 2.5 times higher compared to osteoarthritis of the hip [3].

Traditionally, KOA has been diagnosed by radiography and arthroplasty considered to be the only effective treatment [4]. Recent governmental-approved guidelines for primary care in Denmark and Sweden, however, state that a KOA diagnosis can be made clinically and that the first-line of care is physiotherapy-guided training and education [5]. This is supported through the diagnostic criteria provided by the European League Against Rheumatism (EULAR), developed specifically for primary care [6]. According to EULAR, clinical examination alone can offer a confident diagnosis of KOA. Other diagnostic criteria such as from the American College of Rheumatology (ACR) [7], are primarily developed for hospital care [6].

KOA has long been recognized as a whole organ disease [4], but has more recently been explained as a whole person chronic disease [4]. By the latter understanding, symptoms and signs most often develop slowly over decades [8] and can be manageable for most people through an early diagnosis and individualized strategies [4]. This new paradigm requires a holistic view on diagnosis, clearly linked to selfmanagement aiming to improve the patient's prognosis in the long term.

In view of the International Classification of Function, Disability, and Health [ICF] [9], which represents a systems theory and biopsychosocial understanding $[4,10]$, the diagnostic criteria provided by EULAR and ACR offers limited understanding of the overall clinical situation in patients with KOA. In particular, the criteria do not consider the ICF dimensions activity and participation. Furthermore, similar limitations seem to apply to known risk and prognostic factors documented in systematic reviews [11-13] in which the predominant factors evaluated are summarised mainly into body functions, personal, and diseaserelated factors.

Acknowledging KOA as a whole person disease requires a holistic and biopsychosocial approach. A proper diagnostic assessment needs to include factors derived across the ICF dimensions in order to pinpoint the most important measures for empowering patients to selfmanage and cope with their most valued functional goals $[14,15]$. In a level 1 study of diagnostics, the relevant question is to ask what assessments clearly differ between patients and healthy controls [16]. As indicated by available diagnostic guidelines (EULAR, ACR), as well as evidence from systematic reviews (referred above), that discrimination question has gained little attention in KOA viewed from an overall ICF perspective.

Thus, the main objective of the current study was to explore between-group differences in individuals with KOA and healthy controls, by applying a battery of functional measures derived from guidelines, trial recommendations and previous systematic reviews [17-20] that captures a spectrum of ICF dimensions. The second objective was to examine the rank of these between-group differences (by effect sizes) in order to pinpoint the most deviant functions. The third objective was to analyse how these measures cluster on the ICF dimensions.

\section{Methods}

\section{Design and ethics}

We aimed to perform a cross-sectional, explorative, matched case-control study. The study was approved by the Regional Ethics Committee for Medical and Health Research (REC 2016/984) and was conducted according to the Helsinki declaration. All participants received oral and written information and signed an informed consent form before entering the study. Based on a moderate effect size in knee extension strength between similar groups, i.e. applying unpublished data from our lab related to an earlier study [21], our a priori sample size calculation indicated that we required 20 participants in each group, as the study was allocated $80 \%$ power to detect an effect at $p<0.05$ (cf. Statistics analysis). Although we assumed that no adjustments were needed for multiple comparisons in exploratory studies [22-27], we still aimed for 30 participants in each group. We recruited individuals with KOA referred by general physicians (GPs) to private physiotherapy clinics and to the osteoarthritis school at Trondheim University Hospital, from 
Nov 2016 to Dec 2017. In about the same period, healthy volunteers were recruited via job visits, posters, flyers, and electronic communication, from several different work places in the vicinity of the lab.

Data for each individual participant were collected within a period of approximately 2 weeks through questionnaires and functional tests in the lab. The questionnaires were e-mailed as web-surveys together with the informed consent forms through the Infopad system [28]. The groups were aimed to be frequency-matched [23] on age and gender through the eligibility criteria below. The study was extensive as the questionnaires took on average $40 \mathrm{~min}$ and the physical functional test protocol on average $2.7 \mathrm{~h}$ in the lab. (The current paper presents data from a larger study.) At the very end of the lab session, an accelerometer sensor was applied on the anterior left thigh $[29,30]$ and worn for all hours during 1 week, before it was returned by mail.

\section{Participants eligibility}

The inclusion criteria for patients were having KOA in the tibiofemoral joint(s) diagnosed clinically (by GPs or physiotherapists) and radiologically [31], main problem of pain and limited physical function related to the knee(s), be symptomatic for $>3$ months and daily in the last month, understand Norwegian (orally and written), and be within 45-70 years old. Both genders were included. The upper age-limit was sat to prevent possible confounding due to between-group differences in (i) comorbidity [32] and (ii) physical activity (due to retirement/freedom to reduce activity according to KOA-symptoms), as well as an decline in body function naturally exhibited at high age in both groups [33].

The inclusion criteria for healthy volunteers were aimed to be age and sex frequency-matched to the patients, and able to walk on even ground and negotiate stairs without pain and having no knee complaints.

The exclusion criteria for all participants were surgery to a lower extremity $<3$ years ago, prior lower limb fractures, generalized pain, pain from the spine, hips, or ankles competing with that from the knee, body mass index $(\mathrm{BMI})>35$ [for repeatable optokinematic recordings [34] in the main study, data not reported here], and medical diagnoses other than KOA with clear negative influence on physical function and pain.

\section{Measurements}

The following health status constructs and instruments were implemented, building on prior recommendations and evidence [17-20] and sorted on ICF dimensions. That is, we mainly applied recommended measures from the 2010 Dutch physiotherapy guideline for patients with knee and hip osteoarthritis [17], the Osteoarthritis Research Society International (ORASI) Clinical Trial Recommendations [18], a systematic review on performance-based measures in KOA [19], and measures applied in randomized controlled trials (RCTs) reviewed according to the Cochrane Handbook [20]. Some instruments cross ICF dimensions and are thus presented shortly under more than one ICF dimension. Additional file 1 gives a detailed overview of the measurement properties and scores-of-interpretation of all the applied instruments. Below we present them briefly.

\section{Measures across all the ICF dimensions: body function, activity, and participation}

The Knee Injury and Osteoarthritis Outcome Scale (KOOS), is a freely available knee-specific, self-reporting outcome measure (SROM) for knee-related problems [35]. It measures Pain, Symptoms, Activities in daily living (ADL), Sport and recreation (Sports/Rec), and kneerelated quality of life (QoL) [36, 37]. The scores are converted to percentages, 0 to 100 , worst to best. KOOS includes the Western Ontario and McMaster Universities Osteoarthritis Index (WOMAC) 3.0 to ensure validity for older individuals.

\section{Measures on the ICF body function dimension}

On body function we used 14 measures. Four were mainly performance-based: the Biodex System 4 dynamometer [38, 39] [Biodex System Pro ${ }^{\mathrm{rm}}$, Biodex Medical Systems, NY, USA] for knee extension strength (see Procedures below); the six-minute walk distance test [6MWT] [40] for aerobic endurance; the $30 \mathrm{~s}$ Chair to Stand Test (n30sCST) [ $n=$ number of stands] [40] according to Osteoarthritis Research Society International's (OARSI) video-descriptions [41] for fitness; and the Timed maximum 30 s single-leg stance [T30sSLS] [42] for balance.

Six were pure SROMs: the Numeric Pain Rating Scale (NPRS) [43] for unidimensional pain; two custom-made SROMs for Sleep problems and Vitality (Additional file 1); the KOOS-Pain [44] and -Symptoms [44]; and Tampa Scale of Kinesiophobia (TSK-13) [45] for fear of motion or re-injury. Finally, four were SROMs post performance tests [46]: Borg's Rating of Perceived Exertion Category Ratio 10 (RPE-CR10) and NPRS, both post the 6MWT and the n30sCST.

\section{Measures on the ICF activity dimension}

We used nine measures on the activity dimension. Three were performance-based: the Timed 10-step up-anddown stair climb test [T10StUpDw] [47], the Timed upand-go [TUG] [40] for mobility, balance, walking ability, and fall risk [35], and the 6MWT [47] for long-term walking ability. Three SROMs: KOOS-ADL and -Sports/ Rec for activity problems, and the Patient specific functional scale [PSFS] [48] for the three most problematic activities. Finally, three measures were SROM: the NPRS 
given directly after the T10StUpDw, 6MWT, and the TUG.

\section{Measures on the ICF participation dimension}

We used four measures on the participation dimension. KOOS-QoL for knee-related quality of life [36, 37], the European Health Interview Survey-Quality of Life 8-item index [EUROHIS-QoL] [49] for generic QoL, the Örebro Musculoskeletal Pain Screening Questionnaire 10-items [OMSPQ-10] [50] for work and psychosocial factors, and the AX3 3D accelerometer [3-axis logging accelerometer, Axivity Ltd., Newcastle, UK] $[29,51]$ for time in four intensities of physical activities in daily life (PADL).

\section{Procedures}

Before the lab-session, all participants filled out their respective relevant SROMs. Only the patients, however, registered PSFS and TSK-13. In the lab, within approximately 1 week after the questionnaires, we registered participants' characteristics, degree of radiographic KOA (radiology reports), T30sSLS (on two force plates, Type 9260AA, Kistler, NY, USA), TUG, n30CST, 6MWT, and T10-StUpDw. At the end of the lab session, we measured peak strength (detailed below).

Strength (peak torque) was recorded concentrically for the quadriceps muscle at $60^{\circ} / \mathrm{s}$ of five maximal repetitions applying the passive concentric isokinetic mode [52] of the Biodex ${ }^{\circ}$ System 4 . The participants warmed up by the performance tests 6MWT and 10StUpDw, and a set of 15 repetitions at low-moderate load (in knee extension-flexion). Strength tests were performed with $70^{\circ}$ trunk inclination, all starting with the right side before the left according to the Biodex manual [52]. The passive mode was chosen due to its feasibility in eccentric mode (data not reported here). Fully passive recordings were taken in order to correct for gravity (see Data processing). The system was calibrated before each session. At the very end of the lab-session, an accelerometer was fixed to the participants' left thigh for recording of PADL at $100 \mathrm{~Hz}$ for 1 week.

\section{Data processing}

For T30sSLS data, the time on one leg from the force plates was analyzed using a custom-made algorithm based on inflections in MatLab (v. R2016a, MathWorks Inc., USA). Participants stood with one leg on each force plate and the time on one leg was recorded when a foot left one of the plates. All outputs were validated by inspecting the force graphs using Qualisys Track Manager (QTM, Qualisys AB, Sweden), because the algorithm was invalid for a few recordings where participants stood on one leg already before the start of data registration.

The AX3 accelerometer data was categorized into four different intensities of PADL applying the OmGui Software
[53]. Additional file 1 describes the cut-points for the four PADL-intensity levels.

For knee extensor strength, the passive torques were added to the active ones in order to correct for the limb's own torque. This was done at the $30^{\circ}$ knee-flexed position $\left(0^{\circ}\right.$ was the straight knee) in order to minimize the passive length-tension influence. The result was calculated as best of five repetitions divided by body weight and reported at the $30^{\circ}$ knee flexed position.

\section{Process: recruitment change, age-mismatch, and sensitivity analysis}

Insufficient recruitment from primary physiotherapy care, older patients (able to be) recruited from hospital care, and low recruitment of older healthy volunteers, inflicted a breach in the age-matching. The age-difference necessitated an age-adjusted statistical sensitivity analysis of the parametric data (cf. Statistics below).

\section{Statistics analysis}

Our sample size calculation assumed that no adjustments were needed for multiple comparisons in an exploratory study [22-27] and was performed in the recognized [54] freeware G*Power version 3.1.9.1 [55-57]. This power analysis was an a priori required sample size computation based on t-test (the difference between two independent means), with the following factors: Two tails, $\alpha$ error probability $=0.05, \beta$ error probability $=0.2$ (i.e., power $1-\beta=$ 0.8 or $80 \%$ ), and a moderate effect sized [58] Cohen's $d=$ 0.914 for concentric isokinetic knee extension strength at $60 \%$ s. This required 20 participants in each group. (Additional output parameters were: noncentrality parameter $\delta=2.891$, critical $t=2.024$, degree of freedom $=38$, total sample-size 40 , actual power $=0.804$ ).

For continuous data, normality was inferred by histogram inspections, quantile-quantile plots, and KolmogorovSmirnov tests. For the equal variance assumption, Levene's test was performed. Then, for parametric data with no significant outliers and equal variance, standardized mean difference (SMD) by Cohen's $d$ was calculated with $95 \%$ CI. Independent t-tests were also performed (SPSS v.24, IBM, NY, USA). For continuous non-parametric data, we calculated median differences and 95\% CI (StatsDirect v.2.8.0, Statsdirect Ltd., Cambridge, UK). Then we tested the latter data for differences between groups by medians using Mann-Whitney U (SPSS), before the U statistics and sample size were used to point-estimate Cohen's $d$ applying validated formulas $[59,60]$ at psychometrica.de [61].

Differences between groups on categorical variables were compared by Chi-square test for gender and the Mann-Whitney $U$ test for education and sleep. Then, point-estimates of Cohen's $d$ were calculated via $x^{2}$ and $\mathrm{U}$ statistics, and sample sizes [59-61]. The alpha-level was set to 0.05 for the two-sided tests [22, 24, 25]. 
Data which pertained to both groups, were then compiled and ranked on point-estimates of SMD. The size of the SMD was interpreted according to Hopkins [58] and $p$-value values according to Rosner [62] (Additional file 1). Due to the statistically significant age-difference (see Results), we performed a sensitivity analysis of co-variance (ANCOVA) with age as the covariate on the parametric data.

\section{Qualitative analyses}

For measures with at least moderate effect size, the clustering on ICF dimensions of $(i)$ body function, ( $i i)$ activity, and (iii) participation were based on a content analysis according to the ICF manual [9], definitions of activity as "the ability to move around" [63], and of participation as "the ability to perform daily activities" [64].

\section{Results}

\section{Flow of participants and centres}

Two participants were recruited in physiotherapy clinics, without information on those who declined. At the hospital, we recruited 26 patients out of 36 eligible, where 10 of those invited declined to participate motivated by long traveling distances $(n=3)$, not interested $(n=4)$, afraid of strength testing $(n=2)$, and too time-consuming $(n=1)$. One participant answered the questionnaire but withdrew from the study before the lab-test due to a flareup and was excluded from the analysis. Five individuals did not qualify for participation due to old age $(n=3)$, BMI, and an unstable heart. The 31 healthy control individuals who volunteered represented academic $(n=10)$, administrative $(n=6)$, and health care personnel $(n=7)$. Further, salespersons $(n=3)$, industry employees $(n=3)$, and canteen staff $(n=2)$.

\section{Participant characteristics}

The patients with KOA were on average 6.4 years older. There were no other significant differences on personal factors. On average the patients had had pain for 11 years, had been diagnosed 10 years ago, and showed mostly small-to-moderate radiographic KOA (Table 1).

\section{Main results}

The ANCOVA sensitivity analysis indicated only small bi-directional changes in $p$-values after adjustment for age (Table 2) Thus, we refer to the results of the main analysis below.

Highly significant group differences and very large effect sizes were found for (i) body function on Pain last week, KOOS-Symptoms, and KOOS-Pain; for (ii) activity on KOOS-Sport/Rec and KOOS-ADL; and for (iii) participation on KOOS-QoL and Örebro. Table 3 shows the statistical details and Fig. 1 an overview of the rated effect sizes.
Highly significant group differences and large effect sizes were found for (i) body function post performance on pain-10StepUpDw, pain-CS30s, and pain-6MWT; for (ii) activity on 10StepUpDw; and for (iii) participation on vigorous-intensity PADL (Fig. 1, Table 3).

Significant group differences and moderate effect sizes were found for (i) body function on pain-TUG, knee extensor strength on the involved leg, T30sSLS on the (most) involved leg, and sleep problem; for (ii) activity on 6MWT and TUG; and for (iii) participation on EUROHIS-QoL and moderate-intensity PADL (Fig. 1, Table 3).

\section{Clustering on ICF dimensions}

For measures with at least moderate effect size, we display the clustering on ICF dimensions in the left column of Table 3 (largest to smallest effect size within each level). However, some measures captured more than one dimension: The TUG mobility and 6MWT captured (i) the body function and (ii) activity dimensions, the Örebro-psychosocial (i) and (iii) the participation dimension, whereas the KOOS and EUROHIS-QoL captured all dimensions (i-iii).

\section{Discussion \\ Principle findings}

The main objective of the current study was to explore which recommended/applied measures that most clearly distinguished patients with KOA from healthy controls and describe which ICF dimension those would cluster on. Across 27 measures, the current results indicated that those from the disease specific KOOS (all subscales) and the psychosocial-Örebro questionnaires demonstrated the largest effect sizes for between-group differences, and that these measures together clustered across climbing and the amount of time spent in vigorousintensity physical activity demonstrated the second largest effect size. These clustered on the ICF dimensions activity and participation, respectively. Finally, sleep problems, knee extension strength, static one-leg balance, endurance walking, and Timed up-and-go all showed moderate effect sizes, where the three first clustered on the body function dimension, and the two latter also clustered on the ICF activity dimension.

\section{Results discussion}

\section{ICF body function dimension and KOA}

On the ICF body function dimension, our two measures of pain and the KOOS-Symptom indicated very large effect sizes. This is in accordance with findings from patients awaiting total knee replacement [65]. Further, knee pain is the cardinal complaint in KOA and knee symptoms are central in the diagnostic threes of EULAR [6] and ACR [7]. The result of the current study highlights the importance of a proper baseline pain status, in concordance with the importance of monitoring pain 
Table 1 Personal and health characteristics in the case- and control group

\begin{tabular}{|c|c|c|c|c|c|c|c|}
\hline ICF & Variables & $\begin{array}{l}\text { Cases } \\
(n=28)\end{array}$ & $\begin{array}{l}\text { Controls } \\
(n=31)\end{array}$ & M or Med diff & $\begin{array}{l}\text { M or Med } \\
\text { d or WG } \\
95 \% \mathrm{Cl}\end{array}$ & $\begin{array}{l}\text { Statistics } \\
t, \times 2, U\end{array}$ & $P$-value \\
\hline \multirow[t]{11}{*}{ Personal factors } & Female, n (\%) & $18(64)$ & $16(52)$ & & & $379(x 2)$ & 0.3294 \\
\hline & Age, yrs., M (SD) & $61.7(6.4)$ & $55.3(8.0)$ & 6.4 & $2.6,10.2$ & $3.4(\mathrm{t})$ & $0.0014+$ \\
\hline & Height, m, M (SD) & $1.72(0.10)$ & $1.73(0.09)$ & -0.02 & $-0.07,0.03$ & $-0.7(\mathrm{t})$ & 0.517 \\
\hline & Weight, kg, M (SD) & $82.9(12.7)$ & $80.4(16.6)$ & 2.5 & $-5.2,10.3$ & $0.7(\mathrm{t})$ & 0.517 \\
\hline & $\mathrm{BMI}, \mathrm{kg} / \mathrm{m}^{2}, \mathrm{M}(\mathrm{SD})$ & $24.3(3.5)$ & $25.2(5.1)$ & 1.1 & $-1.1,3.3$ & $1.0(\mathrm{t})$ & 0.317 \\
\hline & Education, n (\%) & & & & & & \\
\hline & secondary school (10 yrs) & $1(4)$ & $0(0)$ & & & & \\
\hline & high school (13 yrs) & $6(21)$ & $6(19)$ & & & & \\
\hline & graduate (16 yrs) & $14(50)$ & $13(42)$ & & & & \\
\hline & post graduate (18yrs. +) & $7(25)$ & $12(39)$ & & & $368(U)$ & 0.281 \\
\hline & Dominant leg (right, left, n) & 26,2 & 28,3 & & & & \\
\hline \multirow{23}{*}{$\begin{array}{l}\text { Patients' body function \& } \\
\text { structure \& activity factors }\end{array}$} & Yrs since diagnosis, M (SD) & $10.2(8.6)$ & & & $6.9,13.6$ & & \\
\hline & Yrs of knee pain, n (\%) & & & & & & \\
\hline & $1 \mathrm{yrs}$ & $2(7)$ & & & & & \\
\hline & 1 to 3 yrs. & $3(11)$ & & & & & \\
\hline & 3 to $10 \mathrm{yrs}$ & $7(25)$ & & & & & \\
\hline & $>10 y r s$ & $16(57)$ & & & & & \\
\hline & Affected knee (n, \%) & & & & & & \\
\hline & One & $14(50)$ & & & & & \\
\hline & Both & $14(50)$ & & & & & \\
\hline & Pain medication ( $n, \%)$ & & & & & & \\
\hline & None & $15(54)$ & & & & & \\
\hline & Paracetamol & $5(18)$ & & & & & \\
\hline & NSAIDs & $5(18)$ & & & & & \\
\hline & Opoids & $2(7)$ & & & & & \\
\hline & Others & $1(4)$ & & & & & \\
\hline & TSK Fear of mov., M (SD) & $24.4(7.7)$ & & & $21.4,27.4$ & & \\
\hline & PSFS Activity 1, Med (IQR) & $3.0(5.0)$ & & & $1.0,5.0$ & & \\
\hline & & \multicolumn{3}{|c|}{ Case-group only } & & & \\
\hline & X-ray grade (n knees, \%) & Inv. leg & Uninv. leg & & & & \\
\hline & No X-rays taken & $0(0)$ & $10(36)$ & & & & \\
\hline & KL-grade ॥ & $9(32)$ & $9(32)$ & & & & \\
\hline & KL-grade III & $17(61)$ & $8(29)$ & & & & \\
\hline & KL-grade IV & $2(7)$ & $1(4)$ & & & & \\
\hline
\end{tabular}

Notes. Case-group = patients with knee osteoarthritis; control-group = individuals without knee complaints; ICF = the International Classification of Function, Disability, and Health of the WHO; $M=$ mean; Med = median; diff = difference between groups; $d=95 \%$ difference between groups; WG = 95\% difference within a group; BMI = body mass index; NSAIDs = Non-Steroid Anti-Inflammatory Drugs; TSK = Tampa Scale of Kinesiophobia; mov. = movement/reinjury; PSFS = PatientSpecific Functional Scale questionnaire; $\mathrm{KL}=$ Kellgren-Lawrence Grade, $\dagger=$ highly significant result

within and between strength exercise sessions shown in methodologically and clinically strong RCTs [66-69]. According to recent systematically reviewed trial-evidence $[20,69]$, pain monitoring is of high value within and between strength-training sessions indicated by the most effective treatment protocols in RCTs also exhibiting high methodological quality [66-69].
The current result indicated a medium between-group difference on knee extension strength. This is comparable to the estimated effect sizes obtained in a metaanalysis of previous cross-sectional case-control studies $[N=6086]$ [70]. Thus, there is ample evidence for patients with KOA demonstrating moderate sized [58] knee extensor weakness compared to healthy controls. 


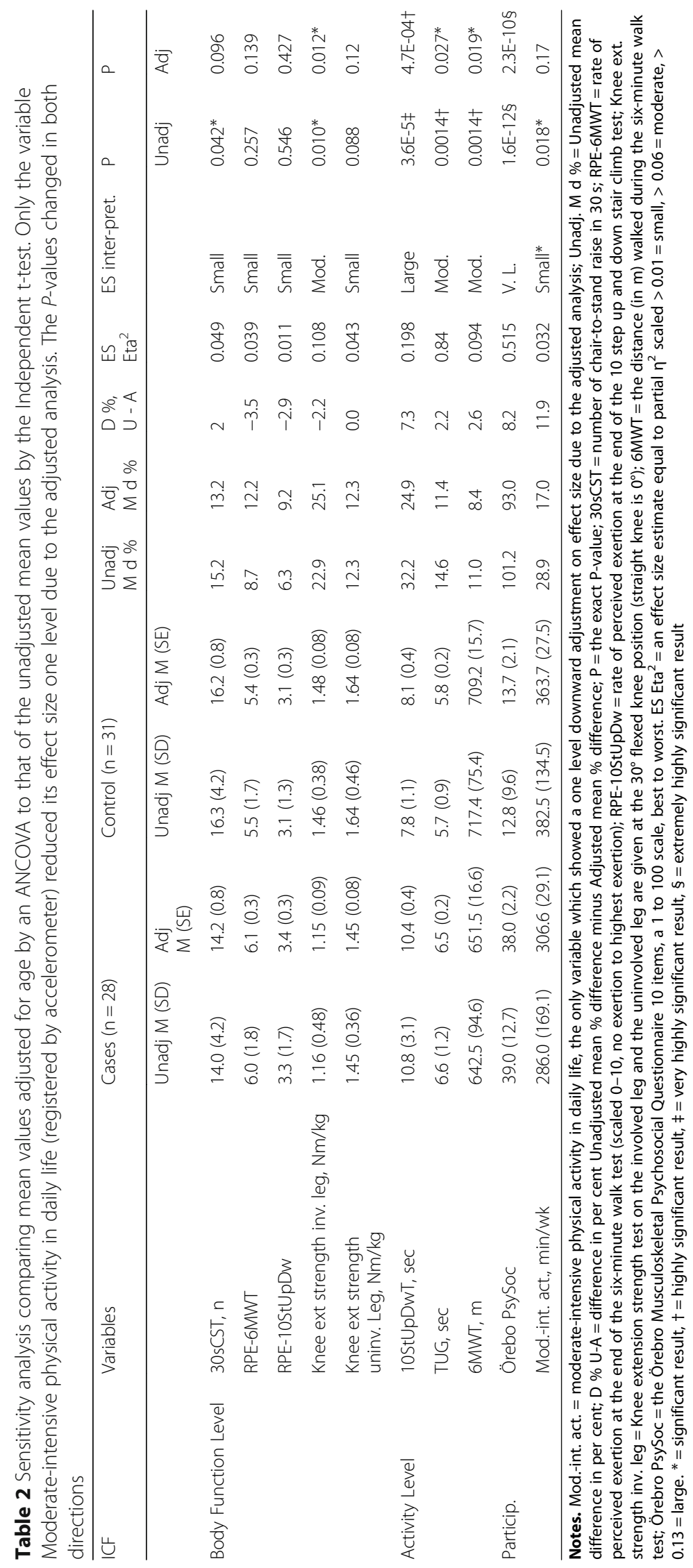




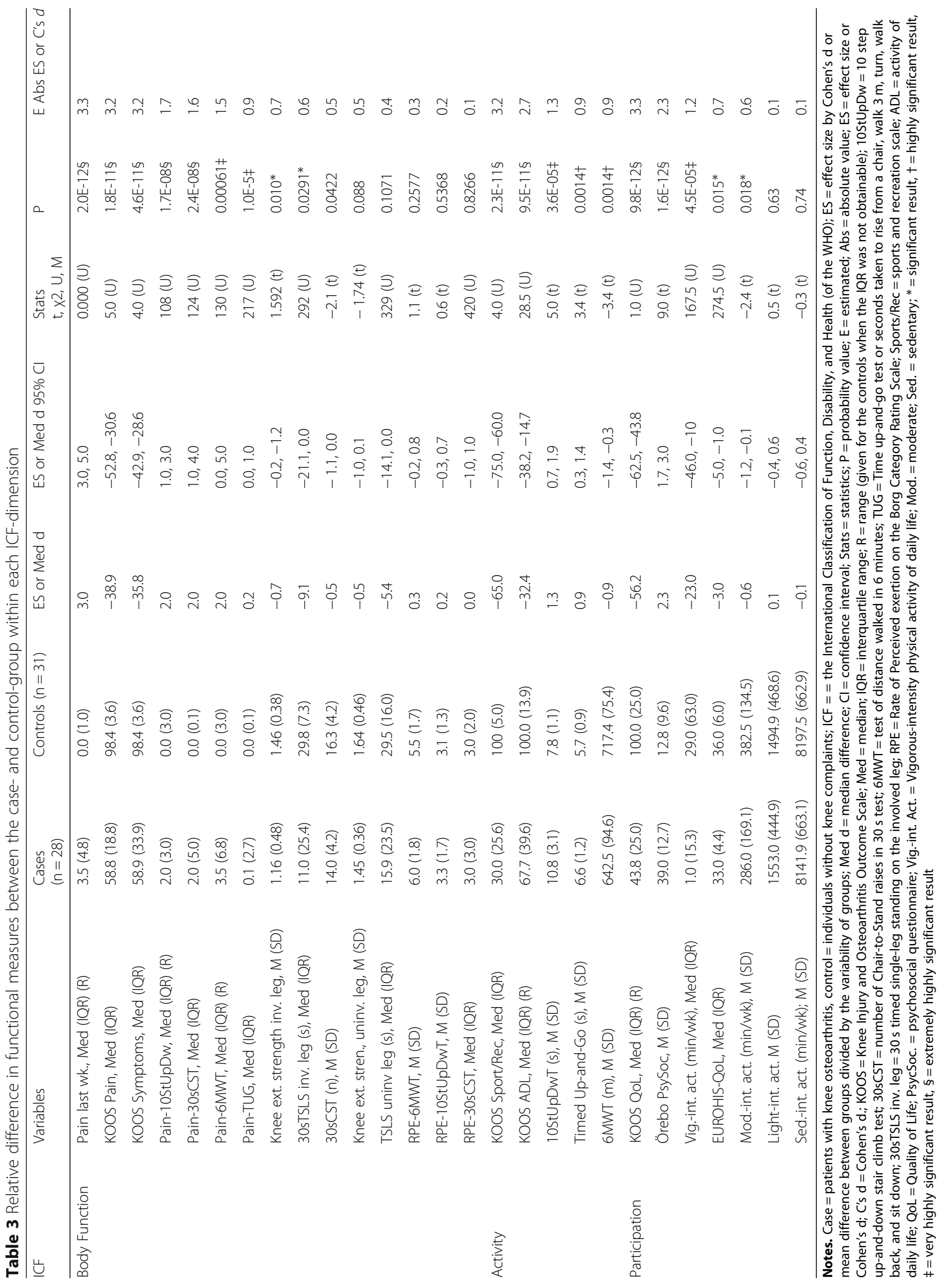




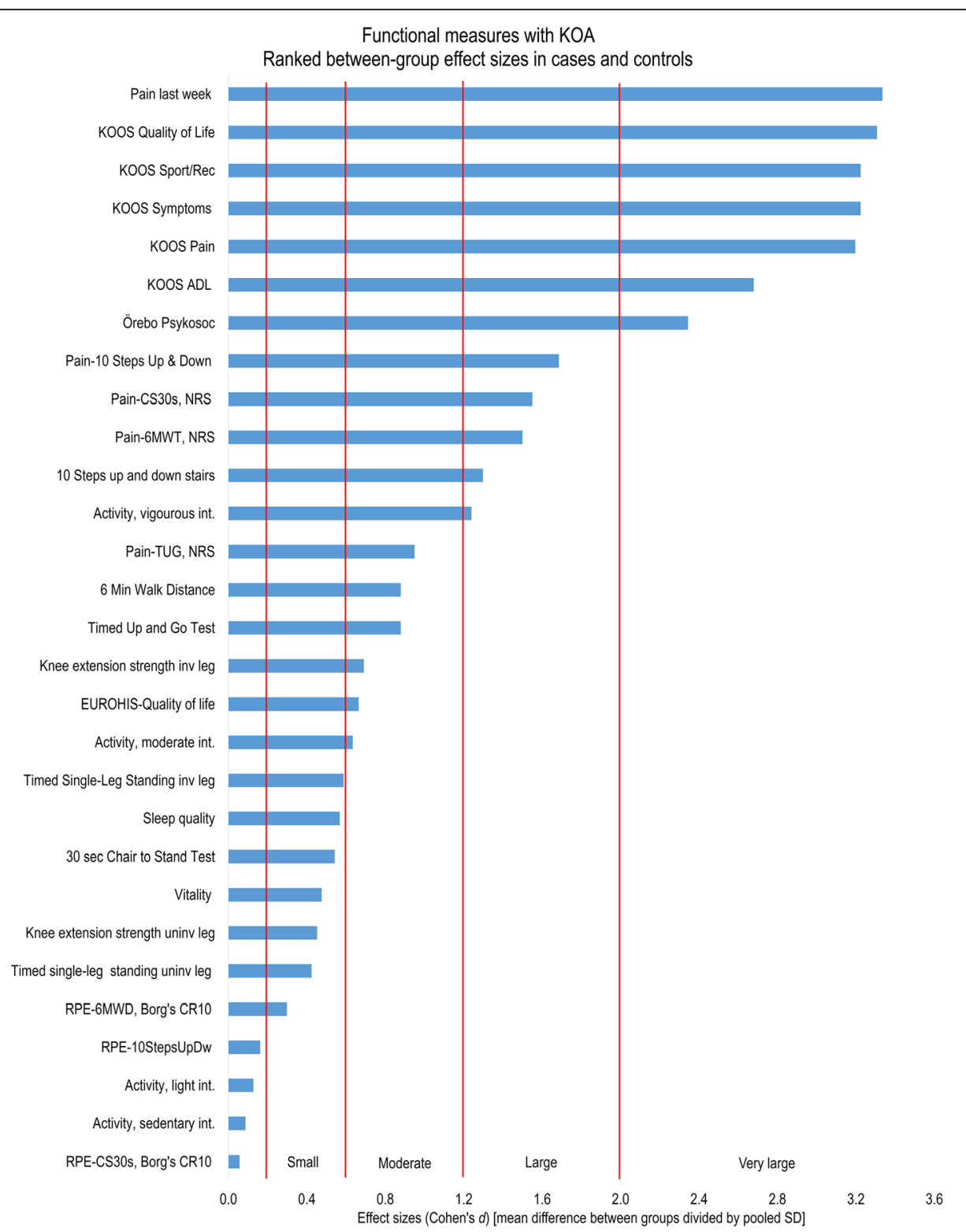

Fig. 1 Relative difference among functional measures in patients with knee osteoarthritis compared to individuals without knee complaints. [Cf. Table 3 and the Result section text for how these measures cluster on the ICF dimension(s)]

Notwithstanding knee extension strength's medium discrimination, the therapeutic importance of knee extensor strengthening is definitely supported by the Ottawa Panel's strongly recommended strength exercise programs (based on a systematic review of RCTs) [69], wherein large effect sizes [58] of treatment have been found for reducing pain and improving function, in particular after three programs of sitting or lying single-leg strength training [66-68]. These three strength programs showed roughly twice the effect sizes $[20,71]$ of comparable recommended programs [72], low risk of bias [20], and PEDro-score $\geq 6$ of 10. Further, their strengthening dose ([repetitions $\times$ resistance $x$ sets]/muscle group) [73] was body-weight independent and objectively recorded [74]. Most importantly though, they coupled pain to dose-response, i.e. linked strength gain to control on pain and objective strengthening dose. Specifically, only these RCTs [66-68] monitored the $24 \mathrm{~h}$ load-pain tolerance in a way similar to that explained for treatment of patients with chronic patellar tendinopathy [75]. Further underpinning the importance of pain vs. dose control, OARSI recommends that strength-training logs also include pain levels [76]. Thus, evidence indicates that knee extensor strength has moderate discrimination whereas the $24 \mathrm{~h}$ load-tolerance measure shows a promising link to large effects on pain, function, and strength of strength exercise therapy. 
The six-minute walk test captures important endurance or long-term walking capability. A relevant question is whether walking only 20 or $40 \mathrm{~m}$, as recommended by ORASI [76], captures the same construct. The present study, however, measured endurance capacity through a six-minute walking test. Herein, the differences between patients with KOA and controls amounted to a moderate sized effect. Our finding is comparable to data from two small-sampled casecontrol studies on patients awaiting total knee replacement [77, 78]. All these findings are, however, superseded by the large effect size in a much larger casecontrol study of patients with moderate KOA $(N=146)$ [79]. This contrast to the latter study is understandable, though, given its $24 \%$ between-group difference in body mass index (vs. our 3\%, 13\% [77], 12\% [78]). In either case, a meta-analysis of clinical trials has just indicated large effects of aerobic endurance training on pain and physical performance [as compared to usual care] [80], and another such analysis indicated clinically relevant effects on physical function of endurance walking applied as a sole intervention [81]. Thus, evidence indicates at least moderate discrimination of the six-minute walk test and that it offers therapeutically important endurance information for effective endurance therapy in patients with KOA.

\section{ICF activity dimension and KOA}

Viewed from the activity dimension of ICF, the present findings on KOOS-ADL and KOOS-Sports/Rec were in concordance with the very large effect size seen in patients awaiting total knee replacement [65] and people with radiographic KOA [82] in case-control studies. Further, in a recent meta-analysis of RCTs [80], strength training and mind-body exercises exhibited the largest therapeutic effects on KOOS-ADL and WOMAC physical function relative to other active therapies that were also compared to standard care. Furthermore, a RCT documented better outcomes when the intervention was a similar (i.e., disease-specific) questionnaire used as a checklist as compared to usual care [83]. Moreover, the above-mentioned trial meta-analysis [80] also indicated large therapeutic effects on pain and physical performance of intensive aerobic endurance exercises. Importantly, such exercises are captured in the problematic activity of the KOOS-Sports/Rec. Thus, evidence indicates important discrimination on KOOS-ADL and KOOS-Sport/Rec, important outcome-measure value of the KOOS-ADL, and finally, that the KOOS-Sports/Rec needs further evaluation as an outcome measure in KOA.

The up-and-down stair climb test demonstrated a large between-group difference in the current study. This is comparable to the $160 \%$ longer average climbingtime for patients with KOA in another similar study [78]. However, the point-estimated effect size in the latter only reached a moderate magnitude, as did the finding in another large case-control study [79]. Indeed, similar-numbered up-and-down stairs climb tests have shown problems of both validity and reliability in musculoskeletal [47] and KOA populations [40]. Increasing and fixing the time of walking upwards to $20 \mathrm{~s}$ and rather record the numbers of negotiated steps thus seems like a promising alternative [40]. Further, stair negotiation as a sole exercise therapy has documented very weak evidence and indeterminable outcomes in a systematic review of RCTs for patients with total knee replacement [84]. Thus, the evidence indicates medium discrimination of a 10 stairs up-and-down climb test with an uncertain connection to effective therapy.

\section{ICF participation dimension and KOA}

On the ICF participation dimension in the present study, the KOOS Quality of life measure amounted to a very large effect size, which is concurrent with that reported in two previous case-control studies [78, 82]. Further, metaanalyses of RCTs indicate important effects of exercise therapy [85] and strength training [80] on health- and knee-related quality of life. Therefore, evidence indicates that the KOOS quality of life measure has very large discriminative value and important outcome measure value in effective trials for patients with KOA.

The current study found a very large between-group difference on the Örebro-psychosocial measure. Surprisingly, no previous case-control studies on KOA appear to have used this questionnaire. Further, according to a systematic review [86], therapy for psychosocial factors might only be of limited additive importance for patients with KOA because no significant effect of psychotherapy on pain was documented. The latter is concurrent with our patients' mild score on kinesiophobia. For the individual patient, however, those scoring above $60 \%$ on the Örebro-psychosocial questionnaire have shown to be of high risk for absenteeism [work/social activities] [87]. Therefore, more studies are needed to challenge the present discriminative ability and evaluate the therapeutic relevance of the Örebro-psychosocial measure in KOA.

When looking for relevant case-control studies to compare the large between-group difference in vigorousintensity physical activity of the present study, we did not find any. Interestingly though, when compared to the 2018 American physical activity guideline for adults [88] which recommends at least $75 \mathrm{~min}$ to $150 \mathrm{~min}$ of vigorous-intensity aerobic physical activity a week, both patients and controls were far from the target (average 0 vs $29 \mathrm{~min}$ ). More relevant perhaps, the alternative of the same guideline is 150 to $300 \mathrm{~min}$ of moderate-intensity physical activity a week, whereupon our patients were seemingly on the target (mean $286 \mathrm{~min}$ ), although the 
variability amongst them was substantial (SD of $169 \mathrm{~min}$ ). Our moderate between-group difference in moderateintensity physical activity, however, agreed with that reported in two such previous studies $[89,90]$. Thus, evidence indicates that objectively obtained levels of moderateintensive physical activity have moderate discrimination and obvious/inherent outcome measure value, but that its therapeutic value needs evaluation in KOA.

\section{Possible implications}

For implications, the most important health measures are those that offer important information on diagnosis/situational understanding, prognosis/therapy, and outcomeevaluation, while being reasonably fast, easily applicable, and low cost. Most of our measures with at least moderate effect sizes might be applicable for such purposes $[6,91]$. More importantly, in several case-control studies, the KOOS has shown very large effect sizes on most factors across all ICF dimensions. Although the KOOS is highly discriminative, cost-free, and disease-specific $[18,35]$, it does not collect frequency and intensity of activities in contrast to for example the generic University of California at Los Angeles activity rating scale [92], the International Physical Activity Questionnaire [93], and the Frenchay Activities Index [35, 94]. For clinicians and researchers, these complementary patient-reported measures are easily available and cost-free [35] or available in web-based computer systems at non-profitable costs (e.g. InfoPad [28] and PROMIS [95]).

Importantly, because only some of the present measures showed substantial discrimination and clear links to effective therapy, we hope the discussion-adjusted conclusions [96] herein raise priority-concerns about which ones to apply clinically or evaluate further in exploratory and confirmative studies [24].

\section{Methods discussion}

The current study has its limitations and strengths. On the one hand, we did not manage to match the groups on age, nor was it possible to adjust for its potential age-inflating functional decline effects on the non-parametric data. Further, the current sample size can be considered rather small if one assumes that adjustments were needed for multiple comparisons in this study according to classical statistical texts [54, 97-100]. On the other hand, the age-adjusted sensitivity analysis of the parametric data supported the unadjusted analysis. Further, both age-means were well within the same clinical middle-aged maturation category (Mesh, PubMed). Yet further, the Results discussion (of the current study) revealed current results in general agreement with those of optimally age-matched confirmative studies. Moreover, for the therein present "inflated" (stair climbing) or "deflated" (endurance walking) results, true result variation among samples $[54,97]$ is perfectly normal also among low risk-of-bias studies (sifted in meta-analyses) [70]. Even further, according to reputative statisticians, there was no need [22-27], or inappropriate or even deleterious to sound statistical inferences [22, 23, 27], to correct for multiple comparisons in the present exploratory study. Additionally, according to the current sample size calculation and the 27 tests, the indicated number of false positive results (type I errors) was $27 \times 0.05=1.35$ [24]. Or, just one of our significant results was most certainly false. Should we thus have adjusted for multiple comparisons? We believe not, because the assumption of such adjustments is the "universal null hypothesis" that holds random variation as the first-order explanation, thus undercutting the premises that nature follows regular laws [22]. And because, in the present study, making no such adjustments kept the statistical power high and type II error-rate low [22, 27]. Concordantly, in the present exploratory low-risk-measures' study, we cared way more about finding true differences than being afraid of accepting a false positive finding. Thus, we infer reasonable internal validity of this study.

The current study weighed its external validity against important considerations. Small-sampled studies [e.g., $n<$ 20] $[54,97]$ are known to increase the risk of chance inflated/deflated effects and thus decrease the generalizability compared to large-sampled studies [e.g., $n>100$ ] [54, 97], whereas both too small or too large samples are unacceptable for clinical, methodological, and ethical reasons [101]. Comparingly, the current study's sample-size was moderate [e.g., $n \geq 20 \leq 100$ ] [54, 97], minimalized according to calculated requirements [with advantages on cost, feasibility, and patients' burden] [101], powered higher than comparable exploratory studies [102, 103], and aligned with the assumption of no need for adjustments of multiple comparisons in exploratory designs [22-27]. Admittingly, the current study limited its generalizability to patients aged $<$ 70 and with $\mathrm{BMI}<35$. The upper age-limit was sat mainly due to the risk of higher comorbidity in the KOA-group at higher ages [32], whereas the upper limit on BMI (including WHO's obesity class I, excluding class II-III) was sat to preserve the repeatability of collected optoelectronic kinematics [34] (data not published here). Comparably, more than 3900 patients with KOA in over 50 Cochrane-reviewed trials were dominated by the middle-aged and aged maturation categories (45-70 years old) with a mean BMI ranging 25 to 32 [72]. Thus, even though an uncertainty remains due to the partly unadjusted age-difference, the external validity of our findings seems substantial. The largest strength of the present study is to show the quantified rank of a plethora of recommended and applied measures in KOA [17-20] whereof only a minority showed considerable between-group differences. The current findings presumably prompt important priority concerns. However, such concerns should at least be influenced by the effects of therapy on these measures (cf. the Results discussion above). 


\section{Conclusions}

In conclusion, among 27 relevant measures, this present study indicates very large differences across all ICF dimensions for the Knee Injury and Osteoarthritis Outcome Scale (KOOS) and the Örebro-psychosocial questionnaire (OMSPQ-10) in patients aged 45-70 with mild to moderate $\mathrm{KOA}$ in a primary/hospital care setting. Clinicians might consider screening by these instruments as means of selecting among relevant supplementary measures demonstrating appropriate discriminative characteristics and clear links to effective therapy. Confirmative studies are needed to further validate these explorative and partly age-unadjusted conclusions.

\section{Supplementary information}

Supplementary information accompanies this paper at https://doi.org/10 1186/s12891-019-2845-0

Additional file 1. Additional methods information regarding measurement instruments, data processing, and statistics applied in Vaarbakken et al. (2019).

\section{Abbreviations}

6MWT: six-minute walk distance test; $\mathrm{AB}$ : Aktiebolag, Swedish for a limited liability company; ACR: American College of Rheumatology; ADL: Activities in daily living; ANCOVA: Analysis of co-variance; AX3 3D accelerometer: A three dimensional or axed accelerometer with the brand name AX3 3D; EULAR: European League Against Rheumatism; EUROHIS-QoL: European Health Interview Survey-Quality of Life 8-item index; GP: general physician; ICF: International Classification of Function, Disability, and Health; KOA: Knee Osteoarthritis; KOOS: Knee Injury and Osteoarthritis Outcome Scale; N: Total number of participants in the meta-analyzed studies or in a single casecontrol study; n30sCST: number of stands in the $30 \mathrm{~s}$ Chair to Stand Test; NPRS: Numeric Pain Rating Scale; OARSI: Osteoarthritis Research Society International; OMSPQ: Örebro Musculoskeletal Pain Screening Questionnaire OMSPQ-10: Örebro Musculoskeletal Pain Screening Questionnaire 10 item version; PADL: physical activities in daily life; PSFS: Patient specific functiona scale; QoL: Knee-related quality of life (in KOOS) or generic quality of life (in the EUROHIS-QoL instrument); QTM: Qualisys Track Manager; REC: Regional Ethics Committee for Medical and Health Research; RPE-CR10: Borg's Rating of Perceived Exertion Category Ratio 10; SMD: Standardized mean difference; Sports/Rec: Sport and recreation (part of KOOS); SPSS: Statistical Package for the Social Sciences, the original name of a statistical software today used in various research areas; SROM: self-reporting outcome measure;

T10StUpDw: Timed 10-step up-and-down stair climb test; T30sSLS: Timed maximum 30 s single-leg stance test; TSK-13: Tampa Scale of Kinesiophobia 13 item version; TUG: Timed up-and-go; WOMAC: Western Ontario and McMaster Universities Osteoarthritis Index

\section{Acknowledgements}

We thank our participants for their time and effort, our scientific-assistants, Erik Borg Kolsung and Anja Liljegren, for co-developing and implementing the Biodex protocol and for test-assistance, our student-assistant Tina Marlen Bråten Mella for the latter, our training- and testing-expert, Dale Reese, for Biodex training, and professor Karin Roeleveld for support with analysis of Biodex data.

\section{Authors' contributions}

AKS developed the main idea for the research design and secured funding for the project. KV further developed the design in cooperation with AKS and KGN. Participants were recruited by KV in cooperation with ME. The digital analysis was performed by $\mathrm{HL}$ (the main responsible), together with $\mathrm{KV}$. The statistical analysis was performed by KV, supervised by HL and AKS $\mathrm{KV}$ wrote the first manuscript drafts. All researchers participated in rewriting the manuscript drafts for scientific content and approved the final version. $\mathrm{KV}$ is the guarantor of the manuscript's authenticity.

\section{Funding}

The project received internal funding from the former Sor-Trondelag University College for a PhD-student position. The funding was granted to A. K. Stensdotter, the head of our project titled "Mechanisms for improved physical function with knee osteoarthritis". There exists no grant number. The funders had no role in study design, data collection and analysis, decision to publish, nor in writing the manuscript.

\section{Availability of data and materials}

The data will be available by reasonable request made to head of project $A$. K. Stensdotter or to the project owner NTNU, Department of Neuromedicine and Movement Science (INB), Program for Physiotherapy.

Ethics approval and consent to participate

This study was approved by the Regional Committees for Medical and Health Research Ethics, Norway (2016/984, the Regional Ethics Committee North) according to the Helsinki Declaration and Norwegian Laws. The participants signed an approved written consent paper before attending the study.

\section{Consent for publication}

Not applicable.

\section{Competing interests}

The authors completed the ICMJE Form for Disclosure of Potential Conflicts of Interest and reported no conflicts of interest with regards to this paper.

\section{Author details}

${ }^{1}$ Department of Neuromedicine and Movement Science, Norwegian University of Science and Technology, Trondheim, Norway. ${ }^{2}$ Faculty of Medicine and Health Sciences, NTNU, Health og Social building, 7491 Trondheim, Norway. ${ }^{3}$ Department of Physical Education and Sport Science, Nord University, Levanger, Norway. ${ }^{4}$ Surgical and Perioperative Sciences, Umea University, Umea, Sweden. ${ }^{5}$ Department of Physiotherapy, Clinic of Clinical Services, Trondheim University Hospital, Trondheim, Norway.

Received: 22 May 2019 Accepted: 20 September 2019

Published online: 22 October 2019

\section{References}

1. March L, Smith EU, Hoy DG, Cross MJ, Sanchez-Riera L, Blyth F, et al. Burden of disability due to musculoskeletal (MSK) disorders. Best Pract Res Clin Rheumatol. 2014;28(3):353-66.

2. Turkiewicz A, Gerhardsson de Verdier M, Engstrom G, Nilsson PM, Mellstrom C, Lohmander LS, et al. Prevalence of knee pain and knee OA in southern Sweden and the proportion that seeks medical care. Rheumatology (Oxford, England). 2015;54(5):827-35

3. Oliveria SA, Felson DT, Reed II, Cirillo PA, Walker AM. Incidence of symptomatic hand, hip, and knee osteoarthritis among patients in a health maintenance organization. Arthritis Rheum. 1995;38(8):1134-41.

4. Roos EM, Arden NK. Strategies for the prevention of knee osteoarthritis. Nat Rev Rheumatol. 2016;12(2):92-101.

5. Sundhedsstyrelsen. Knæartrose - nationale kliniske retningslinjer og faglige visitationsretningslinjer. 1.0 ed. Online: Sundhedsstyrelsen; 2012. p. 88.

6. Zhang W, Doherty M, Peat G, Bierma-Zeinstra MA, Arden NK, Bresnihan B, et al. EULAR evidence-based recommendations for the diagnosis of knee osteoarthritis. Ann Rheum Dis. 2010;69(3):483-9.

7. Altman RD. Criteria for the classification of osteoarthritis of the knee and hip. ScandJRheumatolSuppl. 1987;65:31-9.

8. Thorstensson CA, Andersson ML, Jonsson H, Saxne T, Petersson IF. Natural course of knee osteoarthritis in middle-aged subjects with knee pain: 12year follow-up using clinical and radiographic criteria. Ann Rheum Dis. 2009; 68(12):1890-3

9. World Health Organization W. ICF - International classification of functioning, disability and health. Geneva, Switzerland: WHO; 20012001.

10. Engel GL. The need for a new medical model: a challenge for biomedicine. Science (New York, NY). 1977;196(4286):129-136. 
11. Silverwood V, Blagojevic-Bucknall M, Jinks C, Jordan JL, Protheroe J, Jordan KP. Current evidence on risk factors for knee osteoarthritis in older adults: a systematic review and meta-analysis. Osteoarthr Cartil. 2015;23(4):507-15.

12. Chapple CM, Nicholson H, Baxter GD, Abbott JH. Patient characteristics that predict progression of knee osteoarthritis: a systematic review of prognostic studies. Arthritis Care Res. 2011;63(8):1115-25.

13. de Rooij M, van der Leeden M, Heymans MW, Holla JF, Hakkinen A, Lems WF, et al. Prognosis of pain and physical functioning in patients with knee osteoarthritis: a systematic review and meta-analysis. Arthritis Care Res. 2016;68(4):481-92.

14. Mitchell T, Beales D, Slater H, O'Sullivan P. Musculoskeletal clinical translation framework - from knowing to doing [eBook] 2018. cited 2018. Available from: www.musculoskeletalframework.net.

15. O'Sullivan PB, Caneiro JP, O'Keeffe M, Smith A, Dankaerts W, Fersum K, et al. Cognitive functional therapy: an integrated behavioral approach for the targeted Management of Disabling low Back Pain. Phys Ther. 2018;98(5):408-23.

16. Sackett DL, Haynes RB. The architecture of diagnostic research. Bmj. 2002; 324(7336):539-41.

17. Peter WFH. Update of the Dutch physiotherapy guideline on hip and knee osteoarthritis: Universiteit Leiden, Netherlands; 2010.

18. McAlindon TE, Driban JB, Henrotin Y, Hunter DJ, Jiang GL, Skou ST, et al. OARSI clinical trials recommendations: design, conduct, and reporting of clinical trials for knee osteoarthritis. Osteoarthr Cartil. 2015;23(5):747-60.

19. Dobson F, Hinman RS, Hall M, Terwee CB, Roos EM, Bennell KL. Measurement properties of performance-based measures to assess physical function in hip and knee osteoarthritis: a systematic review. Osteoarthr Cartil. 2012;20(12):1548-62.

20. Fransen M, McConnell S, Harmer AR, Van der Esch M, Simic M, Bennell KL. Exercise for osteoarthritis of the knee: a Cochrane systematic review. $\mathrm{Br}$ J Sports Med. 2015;49(24):1554-7.

21. Gronhaug G, Osteras N, Hagen KB. Quality of hip and knee osteoarthritis management in primary health care in a Norwegian county: a crosssectional survey. BMC Health Serv Res. 2014;14:598.

22. Rothman KJ. No adjustments are needed for multiple comparisons. Epidemiology (Cambridge, Mass). 1990;1(1):43-46.

23. Rothman KJ, Greenland S, Lash TL, Buehler JW, Cahill J, Glymour MM, et al. Modern epidemiology. 3rd ed. ed. Philadelphia: Wolters Kluwer/Lippincott Williams \& Wilkins; 2008.

24. Althouse AD. Adjust for multiple comparisons? It's not that simple. Ann Thorac Surg. 2016;101(5):1644-5.

25. Bender R, Lange S. Adjusting for multiple testing--when and how? J Clin Epidemiol. 2001;54(4):343-9.

26. Nelder J. From statistics to statistical science - Reply. J R Stat Soc Ser D-Stat. 1999;48:269.

27. Perneger TV. What's wrong with Bonferroni adjustments. Bmj. 1998; 316(7139):1236-8.

28. Syltebo R, Odegaard TT. InfoPad AS 2012. Available from: https://infopad.no/.

29. Stewart T, Narayanan A, Hedayatrad L, Neville J, Mackay L, Duncan S. A dual-accelerometer system for classifying physical activity in children and adults. Med Sci Sports Exerc. 2018.

30. Duncan S, Stewart T, Mackay L, Neville J, Narayanan A, Walker C, et al. Wear-Time Compliance with a Dual-Accelerometer System for Capturing 24-h Behavioural Profiles in Children and Adults. Int J Environ Res Public Health. 2018;15:7.

31. Kellgren JH, Lawrence JS. Radiological assessment of osteo-arthrosis. AnnRheumDis. 1957;16(4):494-502.

32. Calders P, Van Ginckel A. Presence of comorbidities and prognosis of clinical symptoms in knee and/or hip osteoarthritis: a systematic review and metaanalysis. Semin Arthritis Rheum. 2018;47(6):805-13.

33. Fulop T, Larbi A, Witkowski JM, McElhaney J, Loeb M, Mitnitski A, et al. Aging, frailty and age-related diseases. Biogerontology. 2010;11(5):547-63.

34. Horsak B, Pobatschnig B, Baca A, Greber-Platzer S, Kreissl A, Nehrer S, et al. Withinassessor reliability and minimal detectable change of gait kinematics in a young obese demographic. Gait Posture. 2017:54:112-8.

35. AbilityLab SR. Rehabilitation Measures Database 2018. Available from: https://www.sralab.org/rehabilitation-measures

36. Roos EM, Roos HP, Lohmander LS, Ekdahl C, Beynnon BD. Knee injury and osteoarthritis outcome score (KOOS)--development of a self-administered outcome measure. The Journal of orthopaedic and sports physical therapy. 1998;28(2):88-96.

37. Roos EM, Toksvig-Larsen S. Knee injury and osteoarthritis outcome score (KOOS) - validation and comparison to the WOMAC in total knee replacement. Health Qual Life Outcomes. 2003;1:17.
38. de Araujo Ribeiro Alvares JB, Rodrigues R, de Azevedo FR, da Silva BG, Pinto RS, Vaz MA, et al. Inter-machine reliability of the Biodex and Cybex isokinetic dynamometers for knee flexor/extensor isometric, concentric and eccentric tests. Physical therapy in sport : official journal of the Association of Chartered Physiotherapists in Sports Medicine. 2015;16(1):59-65.

39. Hartmann A, Knols R, Murer K, de Bruin ED. Reproducibility of an isokinetic strength-testing protocol of the knee and ankle in older adults. Gerontology. 2009;55(3):259-68.

40. Dobson F, Hinman RS, Hall M, Marshall CI, Sayer T, Anderson C, et al. Reliability and measurement error of the osteoarthritis research society international (OARSI) recommended performance-based tests of physical function in people with hip and knee osteoarthritis. Osteoarthr Cartil. 2017;25(11):1792-6.

41. OARSI. Physical Performance Measures 2013. Available from: https://oarsi. org/research/physical-performance-measures.

42. Takacs J, Garland SJ, Carpenter MG, Hunt MA. Validity and reliability of the community balance and mobility scale in individuals with knee osteoarthritis. Phys Ther. 2014;94(6):866-74.

43. Goldsmith ES, Taylor BC, Greer N, Murdoch M, MacDonald R, McKenzie L, et al. Focused evidence review: psychometric properties of patient-reported outcome measures for chronic musculoskeletal pain. J Gen Intern Med. 2018;33(Suppl 1):61-70.

44. Collins NJ, Prinsen CA, Christensen R, Bartels EM, Terwee CB, Roos EM Knee injury and osteoarthritis outcome score (KOOS): systematic review and meta-analysis of measurement properties. Osteoarthr Cartil. 2016.

45. Neblett R, Hartzell MM, Mayer TG, Bradford EM, Gatchel RJ. Establishing clinically meaningful severity levels for the Tampa Scale for Kinesiophobia (TSK-13). Eur J Pain (London, England). 2016;20(5):701-710.

46. Cruz-Almeida Y, Cardoso J, Riley JL 3rd, Goodin B, King CD, Petrov M, et al. Physical performance and movement-evoked pain profiles in community-dwelling individuals at risk for knee osteoarthritis. Exp Gerontol. 2017;98:186-91.

47. Tveter AT, Dagfinrud H, Moseng T, Holm I. Measuring health-related physical fitness in physiotherapy practice: reliability, validity, and feasibility of clinical field tests and a patient-reported measure. The Journal of orthopaedic and sports physical therapy. 2014;44(3):206-16.

48. Chatman AB, Hyams SP, Neel JM, Binkley JM, Stratford PW, Schomberg A, et al. The patient-specific functional scale: measurement properties in patients with knee dysfunction. Phys Ther. 1997;77(8):820-9.

49. Schmidt S, Muhlan H, Power M. The EUROHIS-QOL 8-item index: psychometric results of a cross-cultural field study. Eur J Pub Health. 2006;16(4):420-8.

50. Linton SJ, Nicholas M, MacDonald S. Development of a short form of the Orebro musculoskeletal pain screening questionnaire. Spine (Phila Pa 1976). 2011;36(22):1891-5.

51. Feng $Y$, Wong $C K$, Janeja $V$, Kuber $R$, Mentis HM. Comparison of tri-axial accelerometers step-count accuracy in slow walking conditions. Gait Posture. 2017:53:11-6.

52. Biodex. Biodex Multi-Joint System - Pro Setup/Operation Manual Biodex Medical Systems Inc.; 2014. Available from: http://www.biodex.com/sites/ default/files/850000man_08262revb.pdf.

53. Axivity. AX3 GUl: Axivity Ltd.; 2018. Available from: https://github.com/ digitalinteraction/openmovement/wiki/AX3-GUI.

54. Field A. Discovering statistics using IBM SPSS statistics : and sex and drugs and rock ' $n$ ' roll. 4th ed. ed. SAGE: Los Angeles; 2013.

55. Faul F, Erdfelder E, Buchner A, Lang AG. Statistical power analyses using G*Power 3.1: tests for correlation and regression analyses. Behav Res Methods. 2009:41(4):1149-60.

56. Faul F, Erdfelder E, Lang AG, Buchner A. G*Power 3: a flexible statistical power analysis program for the social, behavioral, and biomedical sciences. Behav Res Methods. 2007:39(2):175-91.

57. Buchner A, Erdfelder E, Faul F, Lang AG. G*Power: Statistical Power Analyses for Windows and Mac Website: Heinrich Heine Universität Düsseldorf 2019. Available from: http://www.psychologie.hhu.de/arbeitsgruppen/allgemeinepsychologie-und-arbeitspsychologie/gpower.html.

58. Hopkins WG. A Scale of Magnitudes for Effect Statistics 2002. Available from: http://www.sportsci.org/resource/stats/effectmag.html.

59. Fritz CO, Morris PE, Richler JJ. Effect size estimates: current use, calculations, and interpretation. J Exp Psychol Gen. 2012;141(1):2-18.

60. Newcombe RG. Confidence intervals for an effect size measure based on the Mann-Whitney statistic. Part 1: general issues and tail-area-based methods. Stat Med. 2006;25(4):543-57. 
61. Lenhard A, Lenhard W. Computation of Effect Sizes 2017. Available from: https://www.psychometrica.de/effect_size.html\#nonparametric.

62. Rosner B. Fundamentals of biostatistics. 8th ed. ed. Boston: Cengage Learning; 2016.

63. Bellamy N, Kirwan J, Boers M, Brooks P, Strand V, Tugwell P, et al. Recommendations for a core set of outcome measures for future phase III clinical trials in knee, hip, and hand osteoarthritis. Consensus development at OMERACT III. J Rheumatol. 1997;24(4):799-802.

64. Terwee CB, Mokkink LB, Steultjens MP, Dekker J. Performance-based methods for measuring the physical function of patients with osteoarthritis of the hip or knee: a systematic review of measurement properties. Rheumatology (Oxford). 2006;45(7):890-902.

65. Naili JE, Esbjornsson AC, Iversen MD, Schwartz MH, Hedstrom M, Hager CK, et al. The impact of symptomatic knee osteoarthritis on overall gait pattern deviations and its association with performance-based measures and patient-reported outcomes. Knee. 2017;24(3):536-46.

66. Huang MH, Lin YS, Yang RC, Lee CL. A comparison of various therapeutic exercises on the functional status of patients with knee osteoarthritis. Semin Arthritis Rheum. 2003;32(6):398-406.

67. Jan $M H$, Lin $C H$, Lin $Y F$, Lin JJ, Lin DH. Effects of weight-bearing versus nonweight-bearing exercise on function, walking speed, and position sense in participants with knee osteoarthritis: a randomized controlled trial. Arch Phys Med Rehabil. 2009;90(6):897-904.

68. Jan MH, Lin JJ, Liau JJ, Lin YF, Lin DH. Investigation of clinical effects of high- and low-resistance training for patients with knee osteoarthritis: a randomized controlled trial. Phys Ther. 2008;88(4):427-36.

69. Brosseau L, Taki J, Desjardins B, Thevenot O, Fransen M, Wells GA, et al. The Ottawa panel clinical practice guidelines for the management of knee osteoarthritis. Part two: strengthening exercise programs. Clin Rehabil. 2017;31(5):596-611.

70. van Tunen JAC, Dell'Isola A, Juhl C, Dekker J, Steultjens M, Thorlund JB, et al. Association of malalignment, muscular dysfunction, proprioception, laxity and abnormal joint loading with tibiofemora knee osteoarthritis - a systematic review and meta-analysis. BMC Musculoskelet Disord. 2018;19(1):273.

71. Young JL, Rhon DI, Cleland JA, Snodgrass SJ. The influence of exercise dosing on outcomes in patients with knee disorders: a systematic review. The Journal of orthopaedic and sports physical therapy. 2018;48(3):146-61.

72. Fransen M, McConnell S, Harmer AR, Van der Esch M, Simic M, Bennell KL. Exercise for osteoarthritis of the knee. The Cochrane database of systematic reviews. 2015;1:Cd004376.

73. Raastad T, Paulsen G, Refsnes PE, Rønnestad BR, Wisnes A. Strength exercise - in theory and praxis. 1th ed. Oslo: Gyldendal Norsk Forlag AS; 20102010.

74. Bartholdy C, Nielsen SM, Warming S, Hunter DJ, Christensen R, Henriksen M. Poor replicability of recommended exercise interventions for knee osteoarthritis: a descriptive analysis of evidence informing current guidelines and recommendations. Osteoarthr Cartil. 2019;27(1):3-22.

75. Malliaras P, Cook J, Purdam C, Rio E. Patellar Tendinopathy: clinical diagnosis, load management, and advice for challenging case presentations. The Journal of orthopaedic and sports physical therapy. 2015;45(11):887-98.

76. Messier SP, Callahan LF, Golightly YM, Keefe FJ. OARSI clinical trials recommendations: design and conduct of clinical trials of lifestyle diet and exercise interventions for osteoarthritis. Osteoarthr Cartil. 2015;23(5):787-97.

77. Thomas AC, Judd DL, Davidson BS, Eckhoff DG, Stevens-Lapsley JE. Quadriceps/hamstrings co-activation increases early after total knee arthroplasty. Knee. 2014;21(6):1115-9.

78. Bade MJ, Kohrt WM, Stevens-Lapsley JE. Outcomes before and after total knee arthroplasty compared to healthy adults. The Journal of orthopaedic and sports physical therapy. 2010;40(9):559-67.

79. Logerstedt DS, Zeni J Jr, Snyder-Mackler L. Sex differences in patients with different stages of knee osteoarthritis. Arch Phys Med Rehabil. 2014:95(12):2376-81.

80. Goh SL, Persson MSM, Stocks J, Hou Y, Welton NJ, Lin J, et al. Relative Efficacy of Different Exercises for Pain, Function, Performance and Quality of Life in Knee and Hip Osteoarthritis: Systematic Review and Network MetaAnalysis. Sports medicine (Auckland, NZ). 2019.

81. Fernandopulle S, Perry M, Manlapaz D, Jayakaran P. Effect of land-based generic physical activity interventions on pain, physical function, and physical performance in hip and knee osteoarthritis: a systematic review and meta-analysis. American journal of physical medicine \& rehabilitation / Association of Academic Physiatrists. 2017:96(11):773-92.
82. Englund M, Roos EM, Lohmander LS. Impact of type of meniscal tear on radiographic and symptomatic knee osteoarthritis: a sixteen-year followup of meniscectomy with matched controls. Arthritis Rheum. 2003:48(8):2178-87.

83. Wikander B, Ekelund P, Milsom I. An evaluation of multidisciplinary intervention governed by functional independence measure (FIMSM) in incontinent stroke patients. Scand J Rehabil Med. 1998;30(1):15-21.

84. Gavin JP, Immins T, Wainwright T. Stair negotiation as a rehabilitation intervention for enhancing recovery following total hip and knee replacement surgery. International journal of orthopaedic and trauma nursing. 2017;25:3-10.

85. Briani RV, Ferreira AS, Pazzinatto MF, Pappas E, De Oliveira SD, Azevedo FM. What interventions can improve quality of life or psychosocial factors of individuals with knee osteoarthritis? A systematic review with meta-analysis of primary outcomes from randomised controlled trials. Br J Sports Med. 2018.

86. Ismail A, Moore C, Alshishani N, Yaseen K, Alshehri MA. Cognitive behavioural therapy and pain coping skills training for osteoarthritis knee pain management: a systematic review. J Phys Ther Sci. 2017;29(12):2228-35.

87. Gabel CP, Burkett B, Melloh M. The shortened Orebro musculoskeletal screening questionnaire: evaluation in a work-injured population. Man Ther. 2013;18(5):378-85

88. Piercy KL, Troiano RP, Ballard RM, Carlson SA, Fulton JE, Galuska DA, et al. The physical activity guidelines for Americans. Jama. 2018;320(19):2020-8.

89. Verlaan L, Bolink SA, Van Laarhoven SN, Lipperts M, Heyligers IC, Grimm B, et al. Accelerometer-based physical activity monitoring in patients with knee osteoarthritis: objective and ambulatory assessment of actual physical activity during daily life circumstances. The open biomedical engineering journal. 2015;9:157-63.

90. Winter CC, Brandes M, Muller C, Schubert T, Ringling M, Hillmann A, et al. Walking ability during daily life in patients with osteoarthritis of the knee or the hip and lumbar spinal stenosis: a cross sectional study. BMC Musculoskelet Disord. 2010;11:233.

91. Dobson F, Bennell K, Hinman R, Roos E, Abbott H, Stratford P, et al. OARSI recommended performance-based tests to assess physical function in osteoarthritis of the hip or knee: authors' reply. Osteoarthr Cartil. 2013; 21(10):1625-6.

92. Terwee $\mathrm{CB}$, Bouwmeester W, van Elsland SL, de Vet $\mathrm{HC}$, Dekker J. Instruments to assess physical activity in patients with osteoarthritis of the hip or knee: a systematic review of measurement properties. Osteoarthr Cartil. 2011;19(6):620-33.

93. Craig CL, Marshall AL, Sjostrom M, Bauman AE, Booth ML, Ainsworth BE, et al. International physical activity questionnaire: 12-country reliability and validity. Med Sci Sports Exerc. 2003;35(8):1381-95.

94. Tse T, Douglas J, Lentin P, Carey L. Measuring participation after stroke: a review of frequently used tools. Arch Phys Med Rehabil. 2013;94(1):177-92.

95. Khanna D, Krishnan E, Dewitt EM, Khanna PP, Spiegel B, Hays RD. The future of measuring patient-reported outcomes in rheumatology: patient-reported outcomes measurement information system (PROMIS). Arthritis Care Res. 2011;63(Suppl 11):S486-90

96. Publication manual of the American Psychological Association. 6th ed. ed. Washington, DC: American Psychological Association; 2010.

97. Altman DG. Practical statistics for medical research. London: Chapman and Hall; 19911991.

98. Tukey JW. Some thoughts on clinical trials, especially problems of multiplicity. Science (New York, NY). 1977;198(4318):679-684.

99. Bland JM, Altman DG. Multiple significance tests: the Bonferroni method. Bmj. 1995;310(6973):170.

100. Ludbrook J. Multiple comparison procedures updated. Clin Exp Pharmacol Physiol. 1998;25(12):1032-7.

101. Rohrig B, du Prel JB, Wachtlin D, Kwiecien R, Blettner M. Sample size calculation in clinical trials: part 13 of a series on evaluation of scientific publications. Deutsches Arzteblatt international. 2010;107(31-32):552-6.

102. Park SK, Kobsar D, Ferber R. Relationship between lower limb muscle strength, self-reported pain and function, and frontal plane gait kinematics in knee osteoarthritis. Clin Biomech (Bristol, Avon). 2016;38:68-74.

103. Dixon J, Howe TE. Quadriceps force generation in patients with osteoarthritis of the knee and asymptomatic participants during patellar tendon reflex reactions: an exploratory cross-sectional study. BMC Musculoskelet Disord. 2005;6:46.

\section{Publisher's Note}

Springer Nature remains neutral with regard to jurisdictional claims in published maps and institutional affiliations. 